\title{
Miranda
}

Revue pluridisciplinaire du monde anglophone /

Multidisciplinary peer-reviewed journal on the English-

speaking world

$10 \mid 2014$

Images on the Move: Circulations and Transfers in film

\section{A travers deux métafilms contemporains : les espaces numériques, nouveaux territoires de l'ontologie cinématographique}

Julien Achemchame

\section{OpenEdition}

Journals

Édition électronique

URL : http://journals.openedition.org/miranda/6302

DOI : $10.4000 /$ miranda.6302

ISSN : 2108-6559

\section{Éditeur}

Université Toulouse - Jean Jaurès

\section{Référence électronique}

Julien Achemchame, "A travers deux métafilms contemporains : les espaces numériques, nouveaux territoires de l'ontologie cinématographique », Miranda [En ligne], 10 | 2014, mis en ligne le 23 février 2015, consulté le 16 février 2021. URL : http://journals.openedition.org/miranda/6302 ; DOI : https:// doi.org/10.4000/miranda.6302

Ce document a été généré automatiquement le 16 février 2021.

\section{cc)}

Miranda is licensed under a Creative Commons Attribution-NonCommercial-NoDerivatives 4.0 International License. 


\title{
A travers deux métafilms contemporains : les espaces numériques, nouveaux territoires de l'ontologie cinématographique
}

\author{
Julien Achemchame
}

1 Inland Empirede David Lynch (2006) et Road To Nowhere de Monte Hellman (2010) apparaissent comme deux œuvres majeures de leurs auteurs, en ce qu'elles sont réflexives et métafilmiques, tout en utilisant les nouvelles technologies numériques. Réflexives, d'une part, parce qu'elles prennent pour objets de leurs récits le tournage d'un film de cinéma, et proposent ainsi toutes sortes d'emboitements narratifs baroques et vertigineux, mais aussi parce qu'elles tissent des liens nombreux, à partir du présent, avec la grande histoire du cinéma, sous forme de citations et reprises. Métafilmiques (Cerisuelo 92-93), d'autre part, parce qu'elles permettent à leurs auteurs tout en dévoilant les coulisses de la production et de la création contemporaine, de s'interroger de manière critique sur son évolution. En ce sens, les films-sommes de ces deux cinéastes cinéphiles apparaissent comme les symptômes d'une prise de conscience de la profonde mutation de leur art et comme une profession de foi cinématographique à l'ère du numérique.

David Lynch et Monte Hellman, cinéastes américains de générations différentes ${ }^{1}$, d'une part parce qu'ils sont peu prolifiques ${ }^{2}$, donnant ainsi à chaque œuvre une importance singulière, d'autre part parce qu'ils ont connu des trajectoires similaires entre centre ${ }^{3}$ et périphérie d'Hollywood ${ }^{4}$ et enfin parce qu'ils admirent la culture européenne ${ }^{5}$, possèdent un regard singulier sur l'industrie cinématographique, entre attirance et répulsion. Ils livrent dans leurs dernières productions, une intéressante réflexion sur le cinéma à l'ère numérique, utilisant chacun à leur façon cette nouvelle technologie (imperfection "poétique» de la Standard Definition pour Lynch contre clarté «illisible» de la High Definition pour Hellman), et réactualisent ainsi une ancienne catégorie de films, le métafilm hollywoodien (né à la fin des années 1920 avec le film 
Show People de King Vidor et théorisé dans les années 2000 par Marc Cerisuelo), qui, dans son essence méta-textuelle même, met en jeu la circulation historique des images tout en se questionnant sur l'origine, le devenir et l'ontologie du cinématographe.

\section{Cinéma en terres numériques : vers un nouveau paradigme ontologique?}

3 Si l'on peut dire que les années 1980-1990 annoncèrent la mort du cinéma et le triomphe (voire la tyrannie) du visuel (i.e. du flux télévisuel), théorisés par Serge Daney dans les colonnes de Libération, les années 2000 furent la décennie du numérique. Révolution majeure qui affecte encore tous les arts, le numérique bouleverse l'industrie cinématographique, comme en son temps l'arrivée du cinéma sonore en 1927, l'émergence de la télévision dans les années 1950 ou bien la démocratisation du magnétoscope et de la vidéo à la fin des années 1970. Ces moments de crises ont, à chaque fois, redessiné les contours de la définition et des usages du terme « cinématographe ». Aussi peut-on s'interroger: en quoi le cinéma est-il soumis à une révolution par l'arrivée du numérique? Qu'inquiète-t-il ?

\section{Une rupture entre l'image et le réel ?}

4 Le passage de l'analogique au numérique, entraînant la disparition de la pellicule photosensible, parce qu'il vient signifier la rupture entre l'image et le réel, redéfinit fondamentalement le réalisme cinématographique, caractéristique ontologique et historique de cet art. Si l'on en croit, en effet, la définition de Jean-Michel Frodon, le cinéma possède trois critères définitoires : d'une part, il est "un art de l'âge de la technique ", d'autre part, il est, "dans sa création et dans sa réception, [...] un art collectif » et enfin, il « est un art de la réalité » (Frodon 33-37), c'est-à-dire qu'il se fonde sur l'enregistrement d'objets préexistant dans le réel. Or, le numérique vient remettre en cause la dernière caractéristique, troisième pilier d'une définition minimale de l'art cinématographique qui lorgne du côté de la théorie ontologique bazinienne évoquant «l'embaumement du temps». Pour Angel Quintana : «Le remplacement du négatif par le disque informatique a mis en crise l'hypothétique vérité des images, en les convertissant en données. L'arrivée du numérique suppose-t-elle la fin du réalisme implicite dans le geste cinématographique, et la transformation du cinéma en un art du virtuel ?» (Quitana 27) Autrement dit, le projet mimétique du cinématographe, comme la croyance en une corrélation entre l'image et le monde, assurée par la médiation de la pellicule photosensible, peuvent apparaître comme remis en cause à l'ère numérique. Qu'attendre désormais du réel lorsque les moyens donnés pour en rendre compte ne peuvent, par essence, s'en approcher que par la modélisation? Ainsi :

Pour E. Couchot, le passage d'une logique de la représentation optique à une logique de la simulation numérique aboutit à un paradoxe évident. Effectivement alors que tout le parcours de l'histoire de l'image se développe autour de l'«adhérence du réel » telle que Roland Barthes l'a définie, le numérique propose une logique dans laquelle la seule réalité préexistant à l'image est une matrice mathématique. Le paradoxe réside dans la capacité du numérique à simuler la représentation, à simuler l'analogique. Le réel que nous montre l'image n'a pourtant plus de référent ni de substrat matériel. (Renard 26)

Ce nouveau statut de l'image entraîne une série de mises en perspective. 


\section{Recréer la fiction cinématographique de l'ère numérique}

6 En effet, le numérique permet de repenser les rapports au sein de la trinité « fiction point de vue - spectateur ». L'artiste, comme le spectateur, sont-ils dépossédés de leurs points de vue par la matrice mathématique substituée au réel? «Avec la numérisation, l'image n'est plus une copie du monde mais une chose hybride et manipulable à l'infini. » (Quintana 47) Elle devient la «trace impossible » d'un monde lointain et pourtant si proche, soumise à la manipulation invisible d'une sorte de Grand Imagier qui ne serait plus le créateur. On peut alors comprendre pourquoi les œuvres de Lynch et d'Hellman, par leurs dispositifs réflexifs et métafilmiques, choisissent d'évoquer, pour l'un, l'histoire d'une actrice perdue lors du tournage du remake d'un film maudit et, pour l'autre, un cinéaste tout aussi perdu lors du tournage d'un film reconstituant un fait divers trompeur. En abordant la question du remake $e^{6}$, uvre qui par essence décentre la perspective auteuriste, et en mettant au premier plan le rôle de l'actrice, mais aussi celui de la spectatrice, au détriment de celui du cinéaste, l'œuvre de Lynch rend sensible les interstices, désormais numériques, entre la fiction, l'instance créatrice et le spectateur. En filmant l'échec créatif d'un cinéaste à re-présenter un réel déjà a priori inatteignable (le fait divers était une tromperie), l'œuvre de Hellman met en lumière les mêmes constats: l'image numérique ne peut rien capter d'un réel inaccessible.

7 De plus, le numérique, support potentiellement sans fin puisque dématérialisé, permet de repenser la relation de l'œuvre avec ses propres limites, fictionnelles comme figuratives : en effet, un film numérique a-t-il vraiment une fin? Les emboîtements narratifs, notamment par l'entremise de la mise en abyme, comme la temporalité cyclique des récits, permettent aux deux métafilms de Lynch et d'Hellman d'être définitivement inachevés et de donner à voir l'espace numérique comme virtuellement infini.

8 Enfin, il faut évoquer également la fragilité de l'image numérique, qui, plus que tout autre image cinémato(-)graphique déjà vouée, par son ontologie, à la disparition, est inquiétée par une transformation virtuelle latente, en autre chose que ce qu'elle semble être. Car le numérique, contaminant tous les arts, se définit d'une part par une esthétique de la simulation mais aussi par une hybridation (Couchot 224), qui a tendance à dissoudre le cinéma hors de ses frontières ${ }^{7}$ et à redéfinir son dispositif de réception (la salle de cinéma). Après avoir fait disparaître son support, le numérique volatilise-t-il aussi son lieu?

9 «Les images ont perdu leur négatif » (Quintana 46). Et cette perte pourrait alors être compensée par la mise en pleine lumière des coulisses de la production cinématographique, montrant ainsi qu'il y a toujours de la création en action, peu importe le support, ou son absence, et en tissant des relations visuelles et mémorielles entre les œuvres à travers l'histoire du cinéma. Car, comme nous l'avons évoqué précédemment, la crise numérique n'est qu'une nouvelle révolution qui donne à lire l'Histoire du cinéma sur le mode dialectique de la linéarité et de la circularité. Parce que le cinéma est dépendant d'une technique en évolution permanente, il n'a cessé, tout au long de son histoire de se réinventer. De l'apparition d'une innovation, obligatoire moment de crise, à son appropriation massive par l'industrie et les cinéastes, le cinématographe a toujours su s'adapter, se renouveler. Ainsi, selon Angel 
Quintana, le cinéma contemporain, contaminé par le numérique, "ne fait que récupérer quelques paramètres de ce cinéma des origines qui furent abandonnés avec le cinéma classique.» (Quintana 16) L'évolution d'une partie du cinéma contemporain marquerait alors un retour aux origines du cinéma, celui de la période 1895-1908, élaborant un "système d'attractions monstratives" (selon la terminologie d'André Gaudreault et Tom Gunning) où chaque plan, dégagé de sa participation au récit d'ensemble de l'œuvre car privilégiant la prouesse technique, forme une unité autonome et un micro-récit. Ce cinéma contemporain, innervé par les innovations technologiques du numérique et de la 3D, effectue un retour aux "attractions", dégagées de toute contingence narrative. Il s'agit alors en partie pour ces films d'exhiber la nouveauté du dispositif technologique et non plus de donner à lire un récit. Cette façon d'appréhender une frange du cinéma actuel permettrait de mettre en évidence la première des deux étapes qui succèdent à l'apparition d'une innovation technique : d'abord une phase d'expérimentation, plutôt libre et désordonnée, avant l'apprivoisement progressif au service de la narration. En effet, dans les années 1910 :

Les courts-métrages [de Charlie Chaplin] élaborèrent une poétique de l'ébauche qui

le conduisit à la perfection dans l'art de la construction du gag. [...]. L'institutionnalisation de Hollywood comme paradis de la fiction classique fit disparaître le cinéma d'improvisation et d'ébauche, le remplaçant par un cinéma de la narration. (Quintana 15)

10 Sur le même modèle, nous pourrions alors mettre en évidence une trajectoire évolutive de la technique numérique entre la phase d'expérimentation lynchienne, puisqu'Inland Empire est tourné en SD entre 2001 et 2006, et l'apprivoisement par Hellman qui réalise Road To Nowhere en HD quatre ans plus tard. Or, l'étude des projets esthétiques des deux cinéastes est bien plus complexe et fascinante.

\section{Inland Empire : retour aux origines du cinématographe et support numérique fantasmé}

11 David Lynch, dès la fin du tournage de Mulholland Drive (2001), n'a cessé de clamer qu'il ne tournerait plus jamais avec de la pellicule $35 \mathrm{~mm}$, préférant le confort proposé par les caméras numériques: légères, simples d'utilisation, offrant un temps de tournage propice au développement de la performance de l'acteur ${ }^{8}$. Délaissant la lourde machinerie cinématographique, le cinéaste a également justifié le choix du caméscope SONY PD150 pour tourner Inland Empire par son envie de ne pas donner à voir des images nettes et parfaites. Parce qu'elles rendent visibles les imperfections, et donc la poésie, du support numérique, les images lynchiennes en SD évoquent un retour aux origines du cinéma, lorsque la technique était neuve et insoumise aux règles classiques $^{10}$. Ce territoire numérique, parce qu'il apparaît vierge, a été une expérience revigorante pour l'esthétique d'un cinéaste en quête d'indépendance créatrice.

\section{Une double filiation hollywoodienne}

12 Ce retour aux origines du cinéma se déploie également dans la dimension intertextuelle des films. Ainsi, Lynch nous propose une étrange reprise d'un extrait du Queen Kelly (1929) d'Erich Von Stroheim tel qu'il apparaît dans son film-fétiche, Sunset Boulevard 
(1950) de Billy Wilder. Cette reprise miroitante d'un intertexte déjà cité mérite que l'on s'y attarde ${ }^{11}$.

Il faut d'abord interroger le choix précis du film d'Eric Von Stroheim via celui de Billy Wilder. Comment ne pas y voir une double filiation esthétique et presque autobiographique de la part de David Lynch? En réanimant les images du grand cinéaste maudit Von Stroheim relues par l'ironie de Wilder, Lynch vampirise un double héritage auteuriste: celui d'un cinéma hollywoodien sans concession à l'égard du système industriel dont il est issu, pétri de culture européenne ${ }^{12}$ et qui porte un regard cynique et radical sur le milieu du spectacle. En effet, on connaît les relations tumultueuses de Von Stroheim avec certains nababs hollywoodiens, Irving Thalberg en tête. Ce dernier mutila les deux chefs-d'œuvre du maître d'origine autrichienne : Foolish Wives en 1922 et Greed, deux ans plus tard. Queen Kelly, avant-dernier projet inachevé de Von Stroheim, connut un sort équivalent : le réalisateur fut évincé en plein tournage par le coproducteur Joseph Kennedy pour des différends liés au dépassement du budget initial et au contenu de scènes jugé trop osé. On connaît également la fameuse réaction de Louis B. Mayer, président alors en fin de règne à la M.G.M., envers Wilder, lors d'une projection-test de Sunset Boulevard en avril 1950: "You bastard! You have disgraced the industry that made you and fed you! You should be tarred and feathered and run out of Hollywood! », à quoi Wilder répondit par un simple «Fuck you » (Lennig 448). David Lynch a subi un traumatisme suite à son expérience sur Dune et sa confrontation avec Dino de Laurentiis. Confronté à la gestion d'une superproduction et dépossédé du final cut, le cinéaste témoigne d'une expérience en partie "horrible». (Lynch, Rodley 92) Grâce à la citation, Lynch se place dans la lignée de ces deux illustres cinéastes, qui, en leur temps, tentèrent de livrer une vision artistique novatrice et sans concession à l'égard d'une institution hollywoodienne oppressante.

Lynch propose également, grâce à cette reprise, une fascinante résurgence d'images primitives à l'ère numérique, captant dans un même mouvement tout autant la crise de la transition du muet vers le parlant, que celle naissante, en 1950, de l'émergence de la télévision, rude concurrente qui va contraindre le cinéma à de multiples innovations techniques et quelques piques dont la célèbre réplique de Norma Desmond : «I am big. It's the screen that got small!» apparaît symptomatique. En réactualisant et en synthétisant ces deux moments critiques, David Lynch commente un état contemporain de l'art cinématographique et entérine une lecture cyclique de l'histoire du cinéma. En faisant le premier métafilm hollywoodien numérique, Lynch démontre que ce type de film naît principalement dans les moments de crises de l'industrie. Subissant les assauts de l'hybridation numérique, l'art cinématographique s'interroge sur son ontologie et sur son devenir, en se replongeant vers son passé et en proposant une circulation renouvelée des images du cinéma.

\section{Une poétique du support numérique}

15 Il faut regarder maintenant de plus près les paramètres de la reprise lynchienne de Sunset Boulevard/Queen Kelly. On remarque ainsi la destruction du cadre originel de l'image, qui mettait en abyme et à distance le dispositif de visionnage, et les ajouts de la couleur comme de la parole, qui de l'écrit anglais se déplace vers le parler polonais, renvoyant ainsi, en les déplaçant, les images des exilés Von Stroheim et Wilder vers une origine est-européenne. Ce transfert culturel inversé donne à voir l'œuvre de Lynch 
comme un miroir relisant, en négatif l'histoire du cinéma hollywoodien. De même, la destruction du cadre, provoquant un mouvement de plongée dans l'image originelle de Von Stroheim, fonctionne à travers un mouvement de déterritorialisation et de tentative de captation d'une émotion archaïque (et cinéphile) originelle. Avec le numérique, tel qu'utilisé ici par David Lynch, le cinéma fait un bond en arrière mais ne perd rien, si ce n'est l'étroitesse de son cadre, et peut ainsi mieux conquérir de nouvelles contrées esthétiques. Cette reprise de l'image double originelle montre la croyance lynchienne en une puissance de l'image numérique qui renoue avec la puissance primitive du cinéma des origines. Ainsi, le choix du gros plan de visage de femme priant dans une chapelle est à ce titre révélateur.

\begin{abstract}
Lynch ne cesse d'explorer les textures dans le but de trouver une beauté nouvelle, expressive, de l'image. INLAND EMPIREnavigue constamment entre le symbolique, le narratif et le plastique. Avec lui, il semble que le numérique confère une nouvelle esthétique aux images et que le support est capable d'amener à une révision des caractéristiques de l'esthétique cinématographique institutionnalisée. [...] Pour Lynch, l'esthétique numérique n'est contraire ni à la réalité, ni à l'art. C'est simplement leur nouvelle alliée. La question clé ne se trouve pas dans la recherche d'un espace pour l'image numérique comme art du virtuel, mais dans la reconceptualisation de la place que les différentes esthétiques du numérique peuvent occuper dans le cinéma contemporain. (Quintana 81-82)
\end{abstract}

Les images lynchiennes en $\mathrm{SD}$, véritables résurgences des premières pellicules instables de l'histoire du cinéma, figurent un nouvel horizon conceptuel qui fantasme une matérialité nouvelle du support cinématographique permettant d'en régénérer les potentialités esthétiques.

\title{
Road To Nowhere : la clarté en haute-définition pour un retour à l'âge d'or hollywoodien
}

Qu'en est-il pour Hellman? Son Road To Nowhere est tourné en 2009 en HD avec l'appareil photo reflex Canon EOS 5D Mark II. Le choix de cette technologie, alors à la pointe de l'innovation, de la part du cinéaste, semble à l'opposé des expérimentations (low def) lynchiennes. Toutefois, la justification proposée par Hellman évoque l'idée d'une évolution à rebours, telle que nous l'avons vue à l'œuvre chez David Lynch. En effet, pour le cinéaste de Road To Nowhere, l'appareil photographique Canon permet une expérience visuelle en tout point comparable à celle du $35 \mathrm{~mm}$. Monte Hellman, aucunement nostalgique du remplacement du support pellicule par son équivalent numérique, postule ainsi une ontologie du cinéma détaché de son support historique : le cinématographe n'a désormais plus besoin de la pellicule pour exister en tant que cinématographe. Cette profession de foi de la part du cinéaste qui conçut la fin inoubliable de Two-Lane Blacktop, prend ici une force particulière.

\section{Une avancée numérique en forme de retour vers l'âge classique hollywoodien}

En montrant l'envers du décor (la production et le tournage d'un film) et l'influence de la révolution numérique sur l'industrie cinématographique hollywoodienne, puisque le cinéaste à l'intérieur du film, Michell Haven, tourne lui-même en numérique, l'œuvre de Hellman réactualise, en 2010, l'ambition réflexive au cœur de tout projet 
métafilmique. Quatre ans après INLAND EMPIRE, Road To Nowhere marque une nouvelle étape de l'évolution du métafilm hollywoodien à l'ère numérique. Mais cette évolution fonctionne, comme pour Lynch, à rebours, car elle s'accompagne d'un retour vers l'âge classique du cinéma. En effet, selon Hellman, le capteur du 5D Mark II correspond à celui d'une caméra $65 \mathrm{~mm}^{13}$. Plus précisément, l'instrument de prises de vue permet un rendu des couleurs semblable à celui du procédé I.B. Technicolor utilisé dans les années $1950-1970^{14}$ et offre ainsi au film une palette de couleurs proches de l'imagerie des années 50, période importante dans l'histoire du métafilm hollywoodien. Ainsi, l'avancée technique s'accompagne d'un retour nostalgique dans le temps cinématographique. À l'instar de tout métafilm, Road To Nowhere apparaît donc comme le symptôme, comme peut l'être sur un autre mode The Artist de Michel Hazanavicius (2011), d'une industrie en crise et/ou en pleine mutation qui s'interroge sur son devenir tout en replongeant vers son passé afin d'en exhumer les mythes. Pourtant le constat de l'apparition de ces nouvelles techniques, ancrant ainsi le film dans une réalité contemporaine, est loin d'être pessimiste et cette ultra-contemporanéité technologique de l'œuvre s'accompagne d'une résurgence de modèles (genres du cinéma classique hollywoodien, citations d'œuvres et d'auteurs majeurs) qui maintiennent l'identité du cinéma et fonctionnent comme autant de repères culturels, narratifs et esthétiques pour le spectateur. Monte Hellman semble, comme David Lynch, revitalisé par les possibilités de ces nouvelles techniques offertes au cinématographe au point de dire à propos de Road To Nowhere: "J'ai l'impression que c'est mon premier film, que tout ce que j'ai fait avant n'était qu'une répétition.» (Hellman, Gaydos 5) Cuvre du renouveau, Road To Nowhere apparaît pour son auteur comme une profession de foi cinématographique empreinte de cinéphilie.

\section{Une profession de foi cinéphilique à l'ère numérique}

19 Comme Lynch, Hellman organise une vaste circulation d'images cinématographiques. Le film est un palimpseste avéré du chef d'œuvre d'Alfred Hitchcock Vertigo (1958), œuvre fétiche de la cinéphilie moderne s'il en est et influence marquante de nombreux cinéastes (Chris Marker, Brian De Palma, Robert Altman, David Lynch, Dario Argento...). Nous pouvons ainsi voir dès le générique du film (dans le film) que l'acteur principal du film de Mitchell Haven s'appelle Cary Stewart, fusion des alter ego hitchcockiens Cary Grant et James Stewart. De manière plus profonde, les histoires de Scottie Ferguson et Mitchell Haven sont en effet semblables: ils sont tous deux hantés par l'image trompeuse d'une femme mystérieuse et attirante, qu'ils retrouvent sans le savoir et qu'ils perdent tragiquement. Transposée dans le milieu du cinéma, et offrant à la Madeleine hitchcockienne l'apparence d'une véritable actrice, l'œuvre de Hellman met en lumière le caractère indubitablement réflexif et métafilmique qui gît au cœur de Vertigo.

En reprenant l'ossature narrative et en proposant une relecture de l'œuvre hitchcockienne à l'ère du numérique, Hellman réactualise une forme nouvelle de cinéphilie, dématérialisée et déterritorialisée, qui prend appui sur une grande circulation des images de cinéma. Le cinéaste Mitchell Haven lance ainsi sur son ordinateur portable le DVD de son propre film et regarde, durant le tournage, sur l'écran plasma de sa chambre d'hôtel ses films-fétiches : The Lady Eve (1941) de Preston Sturges, Le Septième sceau (1957) d'Ingmar Bergman et L'Esprit de la ruche (1973) de Victor Erice. A chaque fois, Hellman organise une plongée dans ces images tantôt 
numériques, tantôt numérisées, signifiant dans le même mouvement leur capacité à phagocyter l'espace cinématographique, soulignant son caractère d'hybridation, et leur pouvoir toujours intact de fascination et d'emprise émotionnelle sur les spectateurs. Ouvrant et clôturant son film sur deux travellings avant lents et longs poussant le spectateur à s'immerger profondément dans les images numériques, Road To Nowhere utilise, jusqu'à la faire éclater, la clarté de la haute définition. Le dernier plan du film, qui en est aussi la mystérieuse affiche, fonctionne en référence au Blow-Up (1967) d'Antonioni. Il s'agit pour le cinéaste de dire qu'il y a toujours un mystère fascinant, une équivoque, qui rend précieuse tout image cinématographique, aussi numérique fûtelle.

Le métafilm, parce qu'il tisse des relations, parce qu'il relie et relit les œuvres cinématographiques et qu'il réfléchit sur ou à partir d'un état contemporain de la production cinématographique, est un lieu privilégié pour voir l'état de la réflexion des artistes sur leur art industriel en voie de mutation. Que nous disent Lynch et Hellman sur l'évolution numérique du cinéma ? Il est intéressant de noter que deux cinéastes de l'ancienne génération acceptent la technologie, l'embrassent même avec joie, y trouvant une nouvelle jeunesse et une régénération de ses potentialités narratives et esthétiques. Il n'y a pas, chez eux, de nostalgie du support pellicule car, à travers leurs dernières œuvres, ils inscrivent la révolution numérique dans une histoire ancienne du cinéma et qui s'appuie sur une circulation toujours plus grande des images de la mémoire cinéphilique.

Toutefois, on peut s'interroger :

La sorte d'introspection filmique du cinéma contemporain serait-elle une marque d'involution, de progrès en arrière? Puisque ce symptôme ne concerne pas, en réalité, tous les films actuels, loin de là, verrait-on se dessiner deux courants? D'une part, tendance «positive» des films dont la vitalité permanente et l'énergie toujours renouvelée leur permettraient d'avancer vers un devenir postcinématographique prometteur. D'autre part, tendance «négative» des films qui, effrayés par la dissolution du cinéma dans les nouveaux media, seraient arc-boutés sur eux-mêmes, cabrés dans une nostalgie interminable ? (Scheinfeigel 22)

Il nous semble que les films de Hellman et Lynch proposent une voie médiane : prenant aux nouvelles technologies la vitalité et l'énergie prometteuses d'images neuves, tout en effectuant un retour vers l'Histoire du cinéma afin de mieux la continuer. Au cœur de ce processus de renouvellement poétique et esthétique la circulation des images cinématographiques constitue un véritable moteur créateur.

\section{BIBLIOGRAPHIE}

Cerisuelo, Marc. Hollywood à l'écran : essai de poétique historique des films : l'exemple des métafilms hollywoodiens. Paris : Presses de la Sorbonne, 2000.

Couchot, Edmond. La technologie dans l'art: de la photographie à la réalité virtuelle. Nîmes : Éditions Jacqueline Chambon, 1998. 
Frodon, Jean-Michel. Horizon Cinéma. L'art du cinéma dans le monde contemporain à l'âge du numérique et de la mondialisation. Paris : Cahiers du cinéma, 2006.

Hellman, Monte. Gaydos, Steven. «Monte Hellman/Steven Gaydos : entretien » in Monte Hellman's Road To Nowhere, dossier de presse. Paris : Capricci, 2011.

Lennig, Arthur. Stroheim, Lexington : The University Press of Kentucky, 2000.

Lynch, David, Rodley, Chris. David Lynch. Entretiens avec Chris Rodley, trad. Serge Grünberg, Charlotte Garson. Paris : Cahiers du cinéma, 2004.

Quintana, Angel. Virtuel ? A l'ère numérique, le cinéma est toujours le plus réaliste des arts, trad. Hélène Fouchard. Paris : Cahiers du cinéma, 2008.

Renard, Caroline. « Numérique le cinéma, et alors ? » In Le Cinéma, et après ? Ed. Maxime Scheinfeigel. Rennes : Presses Universitaires de Rennes, 2010.

Scheinfeigel, Maxime. « Revenir dans les miroirs du cinéma » In Cinergon « Spéculaire ». Ed. JeanPhilippe Trias, $n^{\circ} 19 / 20$ (septembre 2010) : 22 .

Blow-Up. Dir. Michelangelo Antonioni. Bridge Films-Metro-Goldwyn-Mayer. 1967.

Det sjunde inseglet. Dir. Ingmar Bergman. Svensk Filmindustri. 1957.

El espíritu de la colmena. Dir. Victor Erice. Elías Querejeta Producciones Cinematográficas S.L. Jacel Desposito. 1973.

Foolish Wives. Dir. Erich Von Stroheim. Universal Film Manufacturing Company. 1922.

Greed. Dir. Erich Von Stroheim. Metro-Goldwyn Pictures Corporation. 1924.

Inland Empire. Dir. David Lynch. StudioCanal-Asymmetrical Productions. 2006

Queen Kelly. Dir Erich Von Stroheim. Gloria Swanson Pictures. 1929.

Mulholland Drive. Dir David Lynch. Les Films Alain Sarde-Asymmetrical Productions. 2001

Road To Nowhere. Dir. Monte Hellman. Tigers Den Studios. 2010.

Sunset Blvd. Dir. Billy Wilder. Paramount Pictures. 1950.

The Artist. Dir. Michel Hazanavicius. Studio 37 - La Petite Reine - La Classe Américaine - JD Prod France 3 Cinéma - Jouror Productions - uFilm. 2011.

The Lady Eve. Dir. Preston Sturges. Paramount Pictures. 1941.

Vertigo. Dir. Alfred Hitchcock. Paramount Pictures- lfred J. Hitchcock Productions. 1957.

\section{NOTES}

1. Le premier est né en 1946, le second en 1932.

2. Hellman a réalisé 12 longs-métrages entre 1959 et 2010 ; Lynch a réalisé 10 longs-métrages entre 1976 et 2006.

3. Dune (1984) et Two-Lane Blacktop (1971) sont produits par des majors et sont des échecs commerciaux ayant privé leurs auteurs d'une carrière au cœur de l'industrie hollywoodienne, dont ils connaissent les arcanes mais en ont été exclus.

4. Leurs derniers films empruntent d'ailleurs les voies du numérique en partie pour reconquérir leur indépendance. 
5. Hellman a cette belle formule pour qualifier son cinéma : «Mes films viennent d'un endroit au beau milieu de l'Atlantique ». Lynch a tourné une partie d'INLAND EMPIRE en Pologne et a nourri son film du folklore local.

6. Cela permet d'ailleurs d'épingler un état contemporain de l'industrie hollywoodienne qui, depuis de nombreuses années, ne cessent de proposer à ses jeunes spectateurs des remakes.

7. Désormais le cinématographe serait transféré vers les musées et n'aurait plus besoin de la salle de cinéma pour ce constituer en tant que cinématographe. C'est en tout cas la thèse défendue par Luc Vanchéri lorsqu'il évoque le "cinéma contemporain». Voir également sur ce débat le premier chapitre de l'ouvrage de Raymond Bellour, La Querelle des dispositifs, P.O.L., 2012.

8. http://archive.wired.com/culture/lifestyle/news/2007/01/72391?currentPage=2

9. Dernier modèle de caméra Standard Definition fabriqué par SONY avant l'apparition du format HDV en 2004.

10. http://archive.wired.com/culture/lifestyle/news/2007/01/72391?currentPage $=2$ : "The quality is pretty terrible, but I like that. It reminds me of the early days of $35 \mathrm{~mm}$, when there wasn't so much information in the frame or emulsion. [...]. So I love working in digital video. High-def is a little bit too information to me. »

11. http://jurgenfauth.com/wp-content/uploads/wickeddream-6.jpg; http://jurgenfauth.com/ wp-content/uploads/wickeddream-2.jpg

12. Von Stroheim et Wilder sont des exilés d'Europe de l'Est.

13. Procédé de la VistaVision.

14. Il s'agissait d'un processus de teinture qui permettait de ne pas effacer les couleurs.

\section{RÉSUMÉS}

À travers l'exemple de deux œuvres métafilmiques majeures du cinéma américain contemporain, Inland Empire de David Lynch (2006) et Road To Nowhere de Monte Hellman (2010), produites toutes deux à l'aide des nouvelles technologies numériques, nous étudions comment celles-ci témoignent d'un cinéma qui, par la mise en abyme de son dispositif de prise de vue ou de projection et les nombreuses citations intertextuelles, apparaît en quête de sa propre ontologie, à travers la circulation des images. Les deux cinéastes livrent dans leurs derniers opus une réflexion sur le cinéma à l'ère numérique, qui, loin d'annoncer la mort de ce medium, semble être le prélude à un renouvellement esthétique passionnant.

Through the examples of two major metafilmic works of contemporary American cinema, David Lynch's Inland Empire(2006) and Monte Hellman's Road To Nowhere (2010), which have both been produced with new digital technologies, we study how they present an industry of cinema which, through mise en abyme of its own system of representation or projection and many intertextual quotes, appears in search of its own ontology, through the circulation of images. Both filmmakers propose in their last opus a disturbing reflection on cinema in the digital age, which, far from announcing the death of the medium seems to be the prelude to an exciting aesthetic renewal. 
INDEX

Keywords : Inland Empire, Road To Nowhere, metafilm, digital cinema, intertextuality Mots-clés : Inland Empire, métafilm, cinéma numérique, intertextualité

\section{AUTEURS}

\section{JULIEN ACHEMCHAME}

Université Paul Valéry, Montpellier III Chargé d'enseignement, RIRRA21

julien.achemchame@univ-montp3.fr 